\title{
AN EFFICIENT ALGORITHM FOR FINDING MULTIPLE DC SOLUTIONS BASED ON SPICE ORIENTED NEWTON HOMOTOPY METHOD
}

\author{
A.Ushida†, Y. Yamagami†, I. Kinouchi†, Y.Nishio†, Y.Inoue‡ \\ $\dagger$ Department of Electrical and Electronic Engineering, \\ Tokushima University, Tokushima, 770-8506 JAPAN \\ $\ddagger$ Graduate school of Integrated Science and Art, University of East Asia, \\ Simonoseki, Yamaguchi, 751-8503 JAPAN
}

\begin{abstract}
For circuit designing, it is very important to calculate the dc operating points. It is known that if the circuit contains positive feedback loops such as flip-flop and negative resistance circuits, it may happened to have many dc solutions. It is very difficult to find all of the solutions for these circuits. In this paper, we show a very simple Spice oriented Newton homotopy method which can efficiently find out the multiple dc solutions, and perhaps all of the solutions.
\end{abstract}

\section{INTRODUCTION}

Many circuits such as flip-flop, Schmitt-trigger, Hopfield neural, negative resistance circuits [1,2], and so on have multiple dc solutions. There are also many important large scale circuits which contain some of the above sub-circuits $[3,11]$, where a number of the dc solutions may be rapidly increased by the combinations of the solutions from the sub-circuits. In these circuits, it becomes very difficult to find all of the solutions. Therefore, many papers already have been published [3-14]. In the references [3,10,11], nonlinear resistive elements are assumed to be piecewise-linear characteristic. Especially, Yamamura's algorithm [11] can efficiently find all of the solutions. The interval method [12-14] can find all of the solutions of nonlinear algebraic equations in a given accuracy. Although the method is elegant in the mathematical point of view, the computational efficiency is not good for the large scale systems. Note that all of the above methods need to formulate the nonlinear algebraic equations for a given circuit which is a difficult task for large scale circuits in the practice. The others [4-9] are based on the continuation method, which trace the solution curves [4,5], and find the solutions laying on the curves. The homotopy method [8] can trace all of the solutions if we can find out all of the disjoint branches. However, the algorithm to find branches is not so easy when the variables of system are increased. Trajkovic et al.[9] have developed a Spice-like simulator having a globally convergent property which is based on an artificial parameter homotopy method. Inoue $[6,7]$ also has developed a simple Spice oriented simulator which efficiently traces the driving point characteristic curve, where the curve is traced with the transient analysis tool of Spice, efficiently. The method can find out all solutions only if they are laying on the driving point characteristic curve. Thus, there is not yet any practical Spice-like algorithm to find all of the dc solutions.
In this paper, we propose a Newton homotopy method such that

$$
\begin{gathered}
\mathbf{F}(\mathbf{x}, \rho)=\mathbf{f}(\mathbf{x})-(1-\rho)\left[\begin{array}{c}
\mathbf{g}\left(\mathbf{x}_{0}\right) \\
\mathbf{0}
\end{array}\right]=\mathbf{0} \\
\mathbf{f}(\mathbf{x}): R^{n} \mapsto R^{n}, \quad \mathbf{g}\left(\mathbf{x}_{0}\right): R^{n} \mapsto R^{p}
\end{gathered}
$$

where $n$ is the number of nodes, and $p$ is the number of positive feedback loops in the circuit ${ }^{1}$. In our homotopy method, the initial point $(1-\rho) \mathbf{g}\left(\mathbf{x}_{0}\right)$ with $\rho=0$ is corresponding to the input current sources to the specified $p$ nodes, whose sources are continuously changed by a variable $\rho$, and the dc solutions are found at $\rho=1$ on the solution curve. By changing the initial guesses $\mathbf{g}\left(\mathbf{x}_{0}\right)$, we can trace the another solution curves. In this way, it is possible to find the all of the solutions. We developed a user friendly simulator using Spice to implement our Newton homotopy method. From many benchmark examples, we found that our simulator can find all of the solutions on one branch if we choose a suitable initial guess. It has been said that the continuation method is a time-consuming for large scale systems because we need to trace the solution curves in a large dimensional space. However, we found from the theorem in section 2 that we are enough only to consider the solution curves in the $p$-dimensional space. $p$ is usually very small compared to $n$ because it is equal to the number of positive feedback loops. Therefore, the simulator can be usefully applied to the dc analysis of large scale ICs.

\section{NEWTON HOMOTOPY METHOD}

In this section, we consider the ideas of our Newton homotopy method. Let us assume the circuit equation of a nonlinear resistive circuit as follows;

$$
\mathbf{f}(\mathbf{v})=\mathbf{0}, \quad \mathbf{v} \in R^{n}, \quad \mathbf{f}(\cdot): R^{n} \mapsto R^{n}
$$

Applying the Newton homotopy method to (2), we have

$$
\mathbf{F}(\mathbf{v}, \rho)=\mathbf{f}(\mathbf{v})+(\rho-1) \mathbf{f}\left(\mathbf{v}_{0}\right)=\mathbf{0}
$$

where $\rho$ is an additional variable and $\mathbf{v}_{0}$ is an initial guess. Thus, (3) has $n+1$ variables and $n$ equations, so that the

\footnotetext{
${ }^{1}$ We will prove in section 2 that the minimum number of variables to trace the solution curve is equal to the positive feedback loops $p[15,16]$.
} 
solutions of (3) are given by the solution curves in $(n+1)$ space. For simplicity, put $v_{n+1}=\rho$.

Then, we have from (3)

$$
\mathbf{F}(\mathbf{v})=\mathbf{0}, \quad \mathbf{F}(\cdot): R^{n+1} \mapsto R^{n}
$$

Let us trace the curve from the initial point $\mathbf{v}_{0}$ with $v_{n+1}=$ 0 (or $\rho=0$ ). Then, the solutions of (2) are found at the points $v_{n+1}=1$ (or $\rho=1$ ) on the solution curves. In our arclength method, a point $\mathbf{v}$ on the solution curve is described by a function of the distance $s$ from the initial guess $\mathbf{v}_{0}$. It satisfies

$$
(d s)^{2}=\sum_{i=1}^{n+1}\left(d v_{i}\right)^{2}
$$

in the Euclidean space. Combining (5) and (4), we have the following set of algebraic-differential equations:

$$
\begin{aligned}
& F_{1}\left(v_{1}, v_{2}, \ldots, v_{n}, v_{n+1}\right)=0 \\
& F_{2}\left(v_{1}, v_{2}, \ldots, v_{n}, v_{n+1}\right)=0 \\
& F_{n}\left(v_{1}, v_{2}, \ldots, v_{n},, v_{n+1}\right)=0 \\
& \sum_{i=1}^{n+1}\left(\frac{d v_{i}}{d s}\right)^{2}=1
\end{aligned}
$$

It can be solved by the backward-difference method [17], where the derivative of $k$ th order formular at $s=s^{j+1}$ is given by

$$
\left.\frac{d v_{i}}{d s}\right|_{s=s^{j+1}}=\frac{\alpha_{k 0}}{h} v_{i}^{j+1}+Q_{k, i}\left(v_{i}^{j}, \ldots, v_{i}^{j-k+1}\right)
$$

Substituting (7) into (6) at $\mathbf{v}^{j+1}$, the algebraic-differential equations are transformed into the nonlinear algebraic equation as follows:

$$
\left.\begin{array}{c}
F_{1}\left(v_{1}^{j+1}, v_{2}^{j+1}, \ldots, v_{n+1}^{j+1}\right)=0 \\
F_{2}\left(v_{1}^{j+1}, v_{2}^{j+1}, \ldots, v_{n+1}^{j+1}\right)=0 \\
\ldots \ldots \ldots \ldots \ldots \ldots \ldots \ldots \ldots \ldots \ldots \ldots \ldots \ldots \ldots \ldots \ldots \ldots \ldots \ldots \ldots \ldots \ldots \ldots \\
F_{n}\left(v_{1}^{j+1}, v_{2}^{j+1}, \ldots \ldots, v_{n+1}^{j+1}\right)=0 \\
\sum_{i=1}^{n+1}\left\{\alpha_{k 0} v_{i}^{j+1}+Q_{k, i}(\ldots)\right\}^{2}-h^{2}=0
\end{array}\right\}
$$

It is solved by the Newton method

$$
\mathbf{v}^{j+1}=\mathbf{v}^{j}-\left.[\mathbf{J}]^{-1}\right|_{\mathbf{v}=\mathbf{v}^{j}} \mathbf{H}\left(\mathbf{v}^{j}\right)
$$

where $\mathbf{H}\left(\mathbf{v}^{j}\right)$ in (9) is a set of the functions given by (8) with $\mathbf{v}^{j}$ instead of $\mathbf{v}^{j+1}$, and the Jacobian matrix is given by

$$
\mathbf{J}\left(\mathbf{v}^{j}\right)=\left[\begin{array}{cccc}
\frac{\partial F_{1}}{\partial v_{1}} & \frac{\partial F_{1}}{\partial v_{2}} & \ldots & \frac{\partial F_{1}}{\partial v_{n+1}} \\
\frac{\partial F_{2}}{\partial v_{1}} & \frac{\partial F_{2}}{\partial v_{2}} & \ldots & \frac{\partial F_{2}}{\partial v_{n+1}} \\
\vdots & \vdots & & \\
\frac{\partial F_{n}}{\partial v_{1}} & \frac{\partial F_{n}}{\partial v_{2}} & \ldots & \frac{\partial F_{n}}{\partial v_{n+1}} \\
P_{1}\left(v_{1}\right) & P_{2}\left(v_{2}\right) & \ldots & P_{n+1}\left(v_{n+1}\right)
\end{array}\right]_{\mathbf{v}=\mathbf{v}^{j}}
$$

and

$$
P_{i}\left(v_{i}\right)=2 \alpha_{k 0}\left[\alpha_{k 0} v_{i}+Q_{k, i}\left(v_{i}^{j}, \ldots, v_{i}^{j-k+1}\right)\right]
$$

The solution curve can be continuously traced by the above backward-difference method [17] only if the matrix $\mathbf{J}\left(\mathbf{v}^{j}\right)$ is nonsingular on the solution curve. Now, we have the following important theorem about how many and which variables should be chosen in our solution curve tracing algorithm. We call the indispensable variables to trace the solution curve essential variables.

Theorem In our Newton homotopy method, the minimum number of the variables to be chosen is equal to a number of positive feedback loops contained in the circuit, which means that one node voltage in each positive feedback loop should be chosen as the essential variable.

proof The above curve tracing algorithm can trace the solution curve only if the rank of $\mathbf{J}$ is $n+1$. In this case, when the solution curve traces the multiple solutions, it must pass through the turning points $p_{1}$ and/or $p_{2}$ as shown in Fig.1 $[1,15,16,18]$. Hence, it satisfies the relation

$$
\frac{d \rho}{d v_{i}}=0, \text { or } \frac{d v_{n+1}}{d v_{i}}=0
$$

for some $i$ at the turning points.

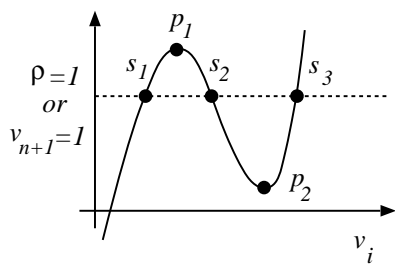

Fig.1 The schematic diagram of a solution curve, $s_{1}, s_{2}, s_{3}$ are solution points, and $p_{1}, p_{2}$ are turning points

Now, differentiating (4) by the arc-length $s$, and applying Cramer's formula, we have

$$
\frac{d \rho}{d s}=\frac{\operatorname{det}\left|\mathbf{J}_{-(n+1),-(n+1)}\right|}{\operatorname{det}\left|\mathbf{J}_{-(n+1),-i}\right|} \frac{d v_{i}}{d s}
$$

Thus, we find from eqs.(11) and (12) that the cofactor $\mathbf{J}_{-(n+1),-i}$ must be nonsingular, but $\mathbf{J}_{-(n+1),-(n+1)}$ is singular. Therefore, if the element $P_{i}\left(v_{i}^{j}\right)$ at $(n+1, i)$ position in $\mathbf{J}\left(\mathbf{v} i^{j}\right)$ is set to a nonzero value, the $(n+1) \times(n+1)$ Jacobian matrix (10) will become nonsingular, and our algorithm can trace the curve at the turning points $p_{1}$ and $p_{2}$. Therefore, we found that the node voltage $v_{i}$ must be chosen as the essential variable.

In the practical Spice simulation, we need to set a probe at the node $i$ to get the voltage $v_{i}$.

Q.E.D.

Corollary A node in positive feedback loop is found in such a manner that when the node voltage at that point is fixed, the characteristic does not have a point satisfying the property given by (11), any more.

From the theorem, our homotopy method can be carried out by solving the following set of algebraic-differential equations:

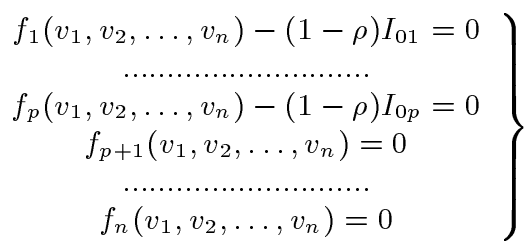

and

$$
\sum_{i=1}^{p}\left(\frac{d v_{i}}{d s}\right)^{2}+\left(\frac{d \rho}{d s}\right)^{2}-1=0
$$

where $\left\{v_{1}, v_{2}, \ldots, v_{p}\right\}$ are the essential variables, and $\left\{I_{01}\right.$, $\left.\ldots, I_{0 p}\right\}$ are the initial guess current sources. 


\section{REALIZATION OF NEWTON HOMOTOPY METHOD}

We have developed our Newton homotopy simulator using Spice as shown in Fig.2.

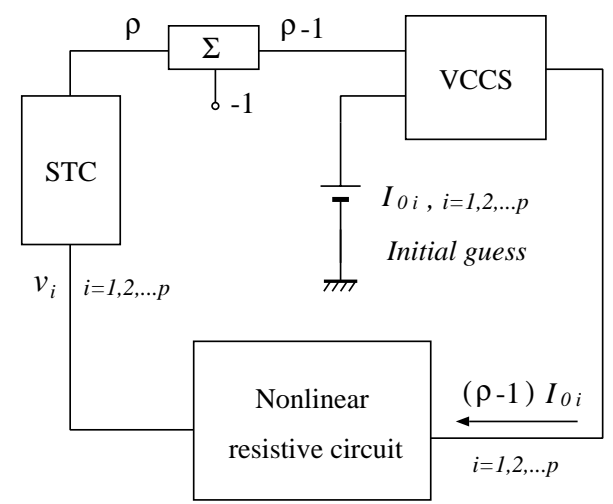

Fig.2 Circuit diagram of our Newton homotopy method

We first set the initial guesses $\left\{I_{01}, \ldots, I_{0 p}\right\}$, and get the input current sources $(\rho-1) I_{0 i}, i=1, \ldots, p$ with VCCSs(voltage-controlled current source) and multipliers. Secondly, the voltage $\rho$ satisfying the relation (14) can be produced by STC(solution curve tracing circuit) [6] as shown in Fig.3.

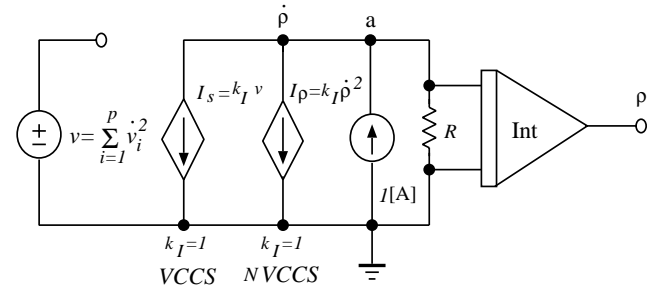

Fig.3 Solution curve tracing circuit (STC)

The input voltages to STC are the essential node voltages $v_{1}, \ldots, v_{p}$ in the positive feedback loops. At first, each voltage is transfered to the corresponding current source by VCCS, so that the STC does not give any disturbance to the nonlinear resistive circuit. The transfered current sources are differentiated by the time $t$ instead of the arc-length $s$ by the use of differentiators or inductor circuits. Thus, we have $\dot{v}_{1}, \ldots, \dot{v}_{p}$, which are again squared to produce $\dot{v}_{1}^{2}, \ldots, \dot{v}_{p}^{2}$ with multipliers, and they are summed by an adder again. Thus, we have

$$
v=\sum_{i=1}^{p} \dot{v}_{i}^{2}
$$

The voltage $v$ is further transformed to the corresponding current source $I_{s}$ as shown in Fig.3. Note that if we assume the node voltage at node "a" is $\dot{\rho}, I_{\rho}=\dot{\rho}^{2}$ is easily produced by a multiplier and a VCCS. Thus, we can realize the circuit satisfying the relation (14). Furthermore, the node voltage $\dot{\rho}$ at "a" is integrated, and we can get $\rho$. Note that $R$ in Fig. 3 is a very large resistance only to avoid the L-J cutset.

\section{ILLUSTRATIVE EXAMPLES}

Large scale circuits usually consist of many sub-circuits having positive feedback loops, such as flip-flop circuit and
Schmitt-trigger circuit, whose sub-circuits have 3 dc solutions, two of them are stable and the other is unstable [18]. Negative resistance circuits [2] have also multiple solutions. If these sub-circuits are coupled in each other, then, they may have many dc solutions. In this section, we solve some important benchmark problems.

\subsection{Transistor-diode circuit}

Fig.4 is a well-known benchmark circuit [3-11] having 11 dc solutions. The circuit consists of 3 Schmitt-trigger circuits. The circuit is constructed in such manner that the output voltages from 2 sub-circuits in the right hand side are inputted into the left hand side circuit through diodes. In this example, we used 3 probes at 3 positive feedback loops as shown in Fig.4. With the initial guess $I_{01}=1[\mathrm{~mA}]$ $I_{02}=1[\mathrm{~mA}] \quad I_{03}=5[\mathrm{~mA}]$, we can trace all of the solutions at $\rho=1$ on the solution curve as shown in Fig.5. We also found that our Newton homotopy method can trace all of the solutions for any initial guess in relatively large region. The solutions are shown in Table 1.

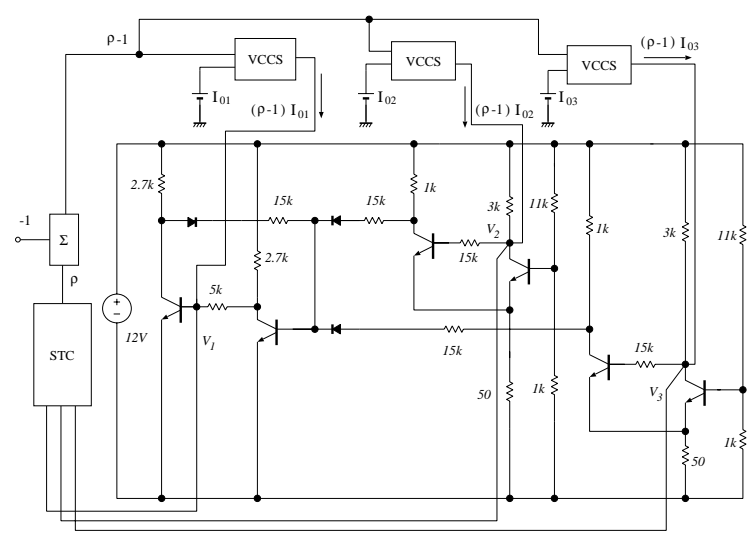

Fig.4 Transistor-diode circuit

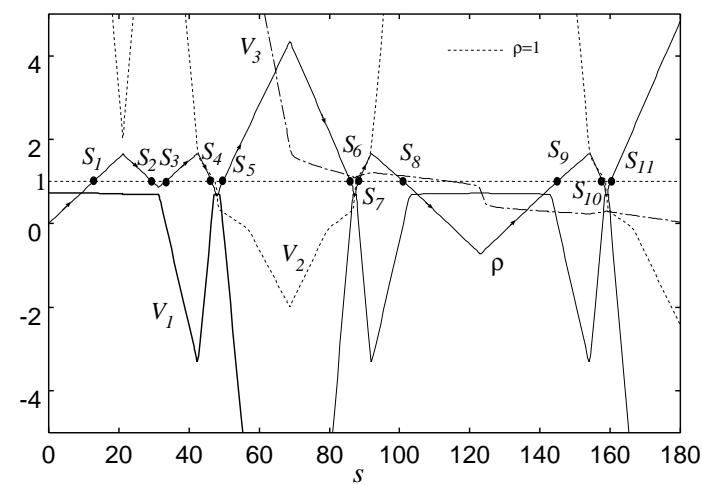

Fig.5 A set of solution curves with our Newton homotopy method, where we get the solutions at $\rho=1$.

\subsection{Flip-flop and Schmitt-trigger circuits}

Let us apply our Newton homotopy method to a circuit shown in Fig.6, where flip-flop and Schmitt-trigger subcircuits are coupled with a resistor $R_{0}$. We found that, for a large $R_{0}$, the two sub-circuits are weakly coupled each other, and it has $9 \mathrm{dc}$ solutions. If we choose the smaller $R_{0}$, the number of solutions will be decreased because of their strong coupling. We need 2 probes at each positive feedback loop as shown in Fig.6. We can trace $9 \mathrm{dc}$ solutions with the 
initial guess $I_{01}=3[m A] \quad I_{02}=5[m A]$ and $R_{0}=2[k \Omega]$. In the strongly coupled circuit with $R_{0}=10[\Omega]$, however, the circuit only has $3 \mathrm{dc}$ solutions.

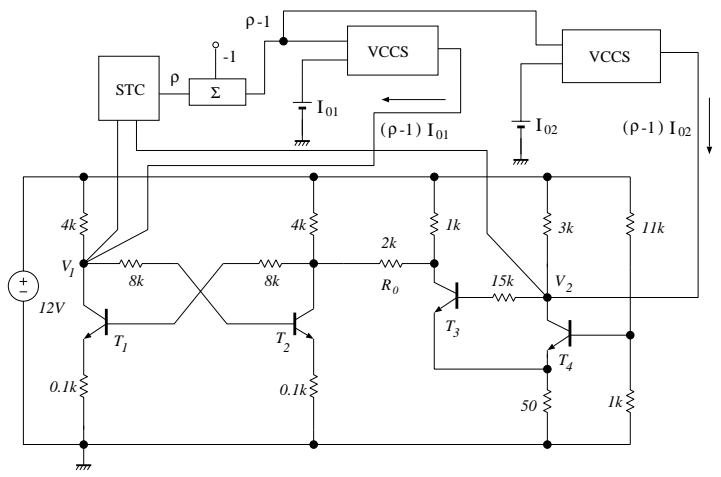

Fig.6 Flip-Schmitt circuit

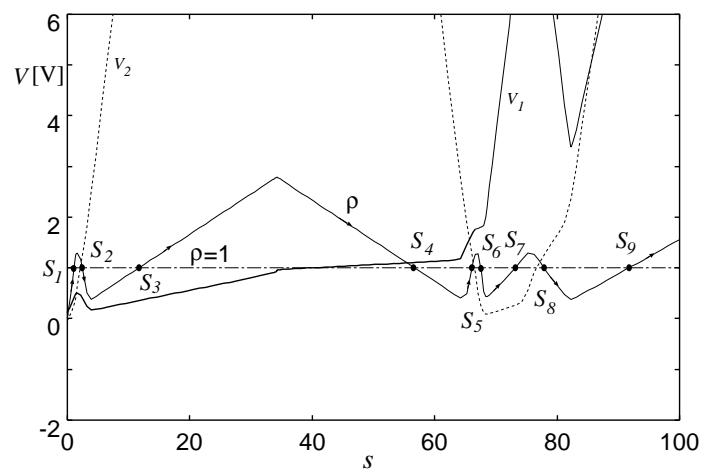

Fig.7 A set of solution curves with our Newton homotopy method, where we get the solutions at $\rho=1$.

Table Dc solutions of transistor-diode circuit

\begin{tabular}{crrr}
\hline Solution & $V_{1}$ & $V_{2}$ & $V_{3}$ \\
\hline & & & \\
$S_{1}$ & 0.719 & 10.222 & 10.222 \\
$S_{2}$ & 0.691 & 10.221 & 10.220 \\
$S_{3}$ & 0.036 & 10.216 & 10.222 \\
$S_{4}$ & 0.028 & 1.134 & 10.223 \\
$S_{5}$ & 0.027 & 0.271 & 10.223 \\
$S_{6}$ & 0.023 & 0.271 & 1.135 \\
$S_{7}$ & 0.024 & 1.135 & 1.135 \\
$S_{8}$ & 0.028 & 10.218 & 1.135 \\
$S_{9}$ & 0.027 & 10.223 & 0.271 \\
$S_{10}$ & 0.023 & 1.134 & 0.271 \\
$S_{11}$ & 0.021 & 0.271 & 0.271 \\
\hline
\end{tabular}

\section{CONCLUSIONS AND REMARKS}

We developed a user friendly simulator to find the multiple dc solutions based on our Newton homotopy method. The simulator is consisted of a simple solution curve tracing circuits(STC), an adder( $\Sigma)$ and voltage controlled current sources(VCCS). The solution curves are traced by the use of transient analysis tool of Spice, and the solutions are found on the curves at $\rho=1$. In order to trace the multiple solutions, we need to set one probe at each positive feedback loop to get the essential node voltage. If we choose a suitable initial guess, it may trace all of the dc solutions. Otherwise, we need to try some curve tracing algorithms from the different initial guesses. Thus, it is possible to find all of the solutions. However, how to find the all of the solutions is the future problem.

\section{REFERENCES}

[1] L.Jiang, Y.Nishio and A.Ushida, "Stability of characteristic curves of nonlinear resistive circuits," IEEE Trans. on Circuits and Systems-I, vol.45, pp.634-643, 1998.

[2] L.O.Chua, J.Yu and Y.Yu, " Bipolar-JFET-MOSFET negative resistance device," IEEE Trans. on Circuits and Systems, vol.CAS-32, pp.46-61, 1985.

[3] M.Tadeussiewicz, "DC analysis of circuits with idealized diodes con sidering reverse bias breakdown phenomenon," IEEE Trans. on Circuits and Systems-I, vol.44, pp.312$326,1997$.

[4] A.Ushida and L.O.Chua, "Tracing solution curves of nonlinear equations with sharp turning points," Int. Jour. of Circuit Theory and Applications,pp.1-21, 1984.

[5] C.W.Yun and K.S.Chao, "Simple solution curves of nonlinear resistive networks," Int. Jour. of Circuit Theory and Applications, vol.11, pp.47-55, 1983.

[6] Y. Inoue "DC analysis of nonlinear circuits using solutiontracing circuits," Trans. of IEICE (A), vo.J74-A, pp.1647$1655,1991$.

[7] Y.Inoue "A practical algorithm for DC operating-point analysis of large scale circuits," Trans. of IEICE (A), vo.J77A, pp.388-398, 1994.

[8] J.Lee and H.-D. Chiang, " Constructive homotopy methods for finding all or multiple dc operating points of nonlinear circuits and systems," IEEE Int. Symp. Circuits and Systems, pp.IV-525-528, 2000.

[9] R.C.Melville, L.Trajkovic, S.-C.Fang and L.T.Watson, "Artificial parameter convergent homotopy methods for the dc operating problems," IEEE Trans. on Computer-Aided Design of Integrated Circuits and Signal Processing, vol.6, pp.861-877, 1993.

[10] T.Ohtsuki, T.Fujisawa and S.Kumagai, "Existence theorems and a solution algorithm for piecewise-linear resistive networks," SIAM J. Math. Anal., vol.8, no.1, pp.69-99, 1977.

[11] K.Yamamura and T.Ohshima, "Finding all solutions of piecewise-linear resistive circuits using linear programing," IEEE Trans. on Circuits and Systems-I, vol.45, pp.434-445, 1998.

[12] G.Alefeld and J.Herzberger, Introduction to Interval computations, New York,NY* Academic, 1983.

[13] L.Kolev and V.Mladenov, "An interval method for finding all operating points of nonlinear resistive circuits," Int.J.Circuit Theory and Applications, vol.18, pp.257-267, 1990.

[14] L.Kolev, "An interval method for global nonlinear analysis," IEEE Trans. on Circuits and Systems-I, vol.47, pp.675-683, 2000.

[15] R.O.Nielsen and A.N.Willson, "A fundamental results concerning the topology of transistor circuits with multiple equilibria," Proc. IEEE vol.68, no.2, pp.196-208, 1980.

[16] T.Nishi and L.O.Chua, "Topological criteria for nonlinear resistive circuits containing controlled sources to have a unique solution," IEEE Trans. on Circuits and Systems, vol.CAS-31, pp.722-741, 1984.

[17] L.O.Chua and P-M Lin, Computer Aided Analysis of Electronic Circuits: Algorithms and Computational Techniques, Prentice-Hall, 1975.

[18] M.M.Green and A.N.Willson, "How to identify unstable operating points,"IEEE Trans. on Circuits and Systemsi-I, vol.CAS-39, pp.820-832, 1992. 